\title{
Equalizing calculus in Trefftz method for solving two-dimensional temperature field of FC-72 flowing along the minichannel
}

\author{
Sylwia Hożejowska • Magdalena Piasecka
}

Received: 13 January 2013/Accepted: 24 February 2014/Published online: 9 March 2014

(c) The Author(s) 2014. This article is published with open access at Springerlink.com

\begin{abstract}
The paper focuses on the numerical solution to two-dimensional temperature field of boiling liquid flowing along a vertical, asymmetrically heated minichannel with a rectangular cross-section. One of the walls of a minichannel is DC supplied single-sided enhanced foil with mini-recesses distributed unevenly in the selected area. The parallel walls are made of glass panes for thermal insulation and they are intended for observation of the two-phase flow and the void fraction. The thin layer of thermosensitive liquid crystal paint on the outer side of the foil enabled to record two-dimensional temperature distribution of outer foil surface. The paper described computations based on Trefftz method for finding two-dimensional temperature field of boiling liquid flowing along the minichannel. The presented research is limited only to the liquid phase of the two-phase mixture observed in the minichannel. The velocity of liquid flowing through the minichannel is represented by a piecewise linear approximating function. To solve energy equation for liquid phase, Trefftz functions specially generated for this purpose were employed. Temperature field in the fluid was approximated by a linear combination of Trefftz functions. Equalizing calculus was applied to the Trefftz method to smooth temperature measurements and reduce measurement errors.
\end{abstract}

S. Hożejowska

Chair of Mathematics, Faculty of Management and Computer Modelling, Kielce University of Technology, al. 1000-lecia P.P.7, 25-314 Kielce, Poland

e-mail: ztpsf@tu.kielce.pl

M. Piasecka $(\bowtie)$

Chair of Mechanics, Faculty of Mechatronics and Machine Building, Kielce University of Technology, al. 1000-lecia P.P.7, 25-314 Kielce, Poland

e-mail: tmpmj@tu.kielce.pl
Temperature at the interface between working fluid and foil amounts to the saturation temperature. Temperature distribution in the foil and the glass pane was also computed using proper Trefftz functions.

\section{List of symbols}

A Section area $\left(\mathrm{m}^{2}\right)$

$b \quad$ Approximation coefficients

c Specific heat $\left(\mathrm{J} \mathrm{kg}^{-1} \mathrm{~K}^{-1}\right)$

hue Component from HSI system, based on hue, saturation, intensity

hue $_{i}$ Hue value corresponding to the set foil temperature

$J \quad$ Functional

$L \quad$ Minichannel length (m)

$m$ The order of polynomial approximating a calibration curve

$n \quad$ Derivative order

$N \quad$ Number of Trefftz functions

$P \quad$ Number of measurement points

$q_{V} \quad$ Volumetric heat flux $\left(\mathrm{W} \mathrm{m}^{-3}\right)$

Re Reynolds number

SEE Standard error estimation (K)

$T$ Temperature (K)

$u \quad$ Velocity $\left(\mathrm{m} \mathrm{s}^{-1}\right)$

$V \quad$ Volume $\left(\mathrm{m}^{3}\right)$

$v \quad$ Trefftz function

$x \quad$ Distance along the minichannel length (m)

$y \quad$ Distance along the glass, foil and minichannel thickness (m)

\section{Greek symbols}

$\alpha \quad$ Heat transfer coefficient $\left(\mathrm{W} \mathrm{m}^{-2} \mathrm{~K}^{-1}\right)$

$\Delta$ Absolute error (accuracy) (K)

$\delta$ Thickness (m) 
$\varepsilon \quad$ Correction $(\mathrm{K})$

$\Phi$ Likelihood function

$\varphi$ Void fraction

$\sigma \quad$ Temperature error (K)

$\lambda$ Thermal conductivity $\left(\mathrm{W} \mathrm{m}{ }^{-1} \mathrm{~K}^{-1}\right.$ )

$\rho$ Density $\left(\mathrm{kg} \mathrm{m}^{-3}\right)$

$\Omega$ Lagrange function

$\omega$ Lagrange multiplier

\section{Subscripts}

$\begin{array}{ll}\text { ave } & \text { Average } \\ G & \text { Glass } \\ F & \text { Foil } \\ i & \text { Measurement point } \\ \text { in } & \text { Inlet } \\ L & \text { Liquid } \\ M & \text { Minichannel } \\ \text { max } & \text { Maximal value } \\ \text { out } & \text { Outlet } \\ \text { sat } & \text { Saturation } \\ v & \text { Vapour }\end{array}$

\section{Superscript}

corr Corrected

\section{Introduction}

The development of the high-tech integrated circuits technology involves high power consumption and related heat generation; hence the cooling systems for microelectronic chips face the challenge of heat dissipation in confining spaces. Flow boiling in minichannels is an option because high heat transfer rate and minichannels are ideal for these applications due to their compactness and low pressure drop. Yet, these issues have not been studied enough. The research of the enhanced structures is increasingly attractive due to their theoretical enhancement potential for heat transfer. The summary of relevant literature focused on heat transfer in rectangular minichannels is presented in [1], and in addition, having enhanced surfaces - in [2, 3]. Trefftz method used for solving issues related to heat transfer in minichannels with new Trefftz functions is employed for smoothing measurement data and reducing measurement errors.

\section{Experimental research}

\subsection{Experimental stand}

The area of interest is the issue of heat transfer in cooling liquid flowing through a rectangular minichannel with one uniformly heated enhanced wall. The flow system was

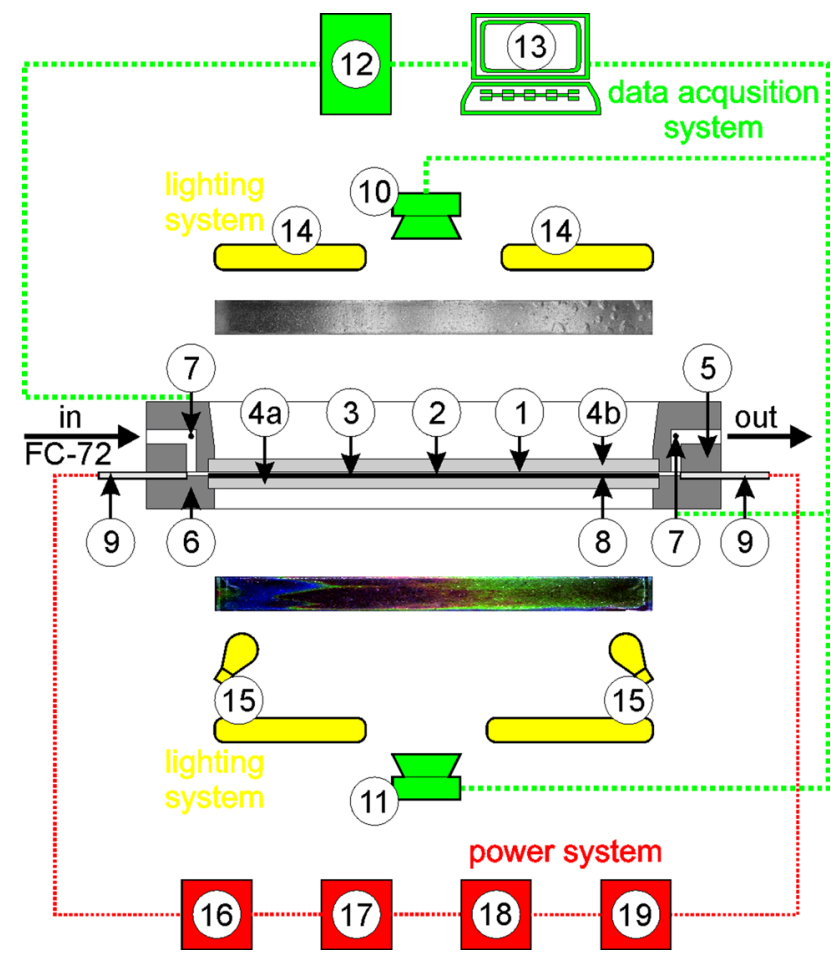

Fig. 1 The schematic diagrams of the most elements of the experimental stand: \#1-minichannel, \#2-heating foil, \#3-liquid crystal layer, \#4a, b-glass pane, \#5-channel body, \#6-front cover, \#7thermocouple, \#8-enhanced side of the foil, \#9-copper element \#10digital SLR camera, \#11-digital camera, \#12-data acquisition station, \#13-a laptop, \#14-halogen reflectors, \#15-fluorescent and LED lamps, \#16-inverter welder, \#17-shunt, \#18-ammeter, \#19-voltmeter

discussed in [1-5]. The essential part of the experimental stand is the test section with a minichannel (Fig. 1, \#1). It is $1 \mathrm{~mm}$ deep, $40 \mathrm{~mm}$ wide and $360 \mathrm{~mm}$ long, oriented vertically. The heating element for the working fluid (FC-72) flowing along the minichannel is alloy foil (\#3) stretched between the front cover (\#6) and the channel body (\#5). This thin foil (approx. $0.1 \mathrm{~mm}$ ) is enhanced on the side of the fluid flowing in the minichannel. It is energized by copper elements (\#9) with direct current of controlled intensity. It is possible to observe both surfaces of the minichannel through two openings covered with glass panes. One pane (\#4a) allows observing changes in the temperature of the foil surface. It is a plain side of the heating foil (between the foil and the glass), covered with thermosensitive liquid crystal paint (\#3). The opposite surface of the minichannel (from the enhanced side of the heating foil) is observed through the other glass plane (\#4b), which helps recognize the two-phase flow patterns. K-type thermocouples and two pressure converters (\#7) are installed in the inlet and outlet of the minichannel. The data sources from the experiment and image acquisition system include: \#10-Canon Eos 550D digital SLR camera, \#11-Canon G11 digital camera, \#14-two $1 \mathrm{~kW}$ halogen reflectors with forced air cooling and heat resistant 
(a)

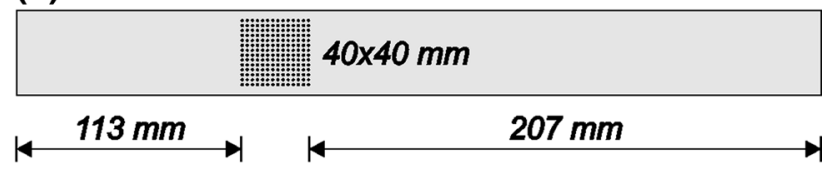

(b)

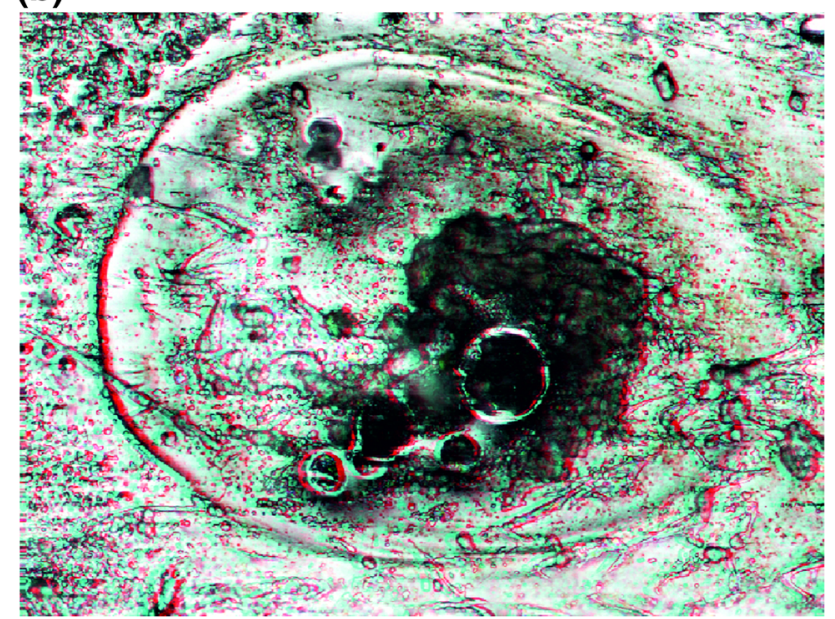

(c)

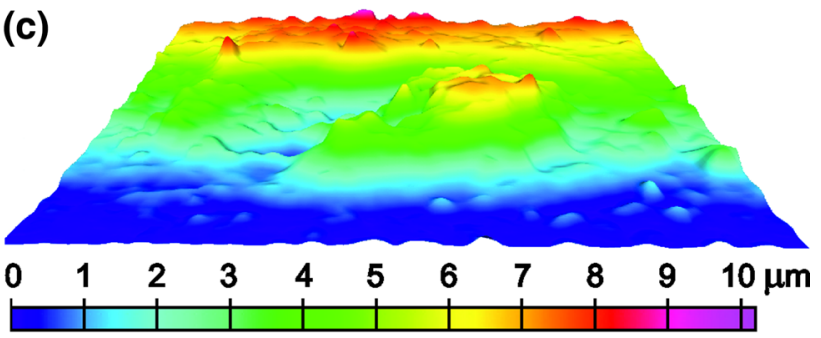

Fig. 2 The scheme of enhanced foil with mini-recesses a with photo b and 3-d topography $\mathbf{c}$ of the sample area

casing, and \#15-fluorescent and LED lamps emitting "cool white light". Experimental data have been recorded with DaqBoard 2005 data acquisition station (\#12) equipped with DASYLab software installed on a laptop (\#13). The heating surface in the minichannel is provided with electric current by an inverter welder (\#16) as current regulated DC power supply (up to $300 \mathrm{~A}$ ). On the side of the foil contacting the fluid in the minichannel, mini-recesses were distributed unevenly in the selected area of the foil $(40 \times 40 \mathrm{~mm})$ as shown in Fig. 2a. The recesses were obtained by spark erosion. The layer of melted metal of the foil and the electrode material, a few $\mu \mathrm{m}$ high, reaching locally $5 \mu \mathrm{m}$, accumulates around the cavities. The depth of the craters of cavities is usually below $1 \mu \mathrm{m}$. The photographs of the plain and enhanced foil is shown in Fig. 2b, c.

\subsection{Experimental methodology}

Application of liquid crystals for the detection of twodimensional heating surface temperature distribution must be preceded by colour (hue) temperature calibration [1].

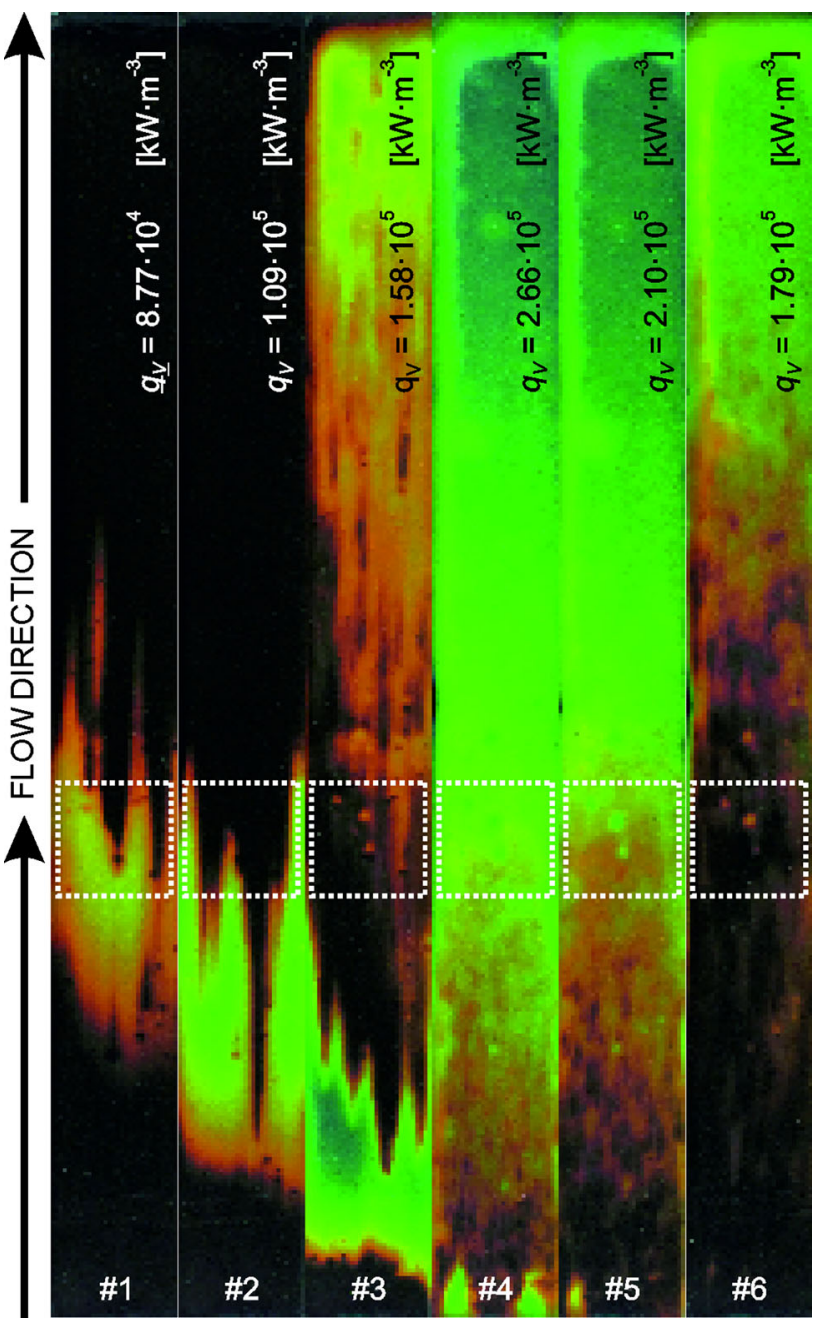

Fig. 3 Colour heating foil images while increasing and later decreasing heat flux supplied to the foil, experimental parameters: flow velocity $0.17 \mathrm{~m} / \mathrm{s}$, mass flux $285 \mathrm{~kg} /\left(\mathrm{m}^{2} \mathrm{~s}\right)$, Reynolds number $R e=880$, inlet pressure $125 \mathrm{kPa}$, inlet liquid subcooling $42 \mathrm{~K}$, dotted lines show location of mini-recesses

FC-72 working fluid, at the temperature below its boiling point, flows laminarly into the minichannel (\#1, Fig. 1). The gradual increase in the electric power supplied to the heating foil results in an increased heat flux transferred to the liquid in the minichannel. The current supplied via copper elements (\#9) to the heating foil (\#2) is controlled by an electrical system equipped with an inverter welder (\#16). This leads to the incipience and next to the development of nucleate boiling. Then, the current supplied to the foil reduces gradually. Thanks to the liquid crystal layer located on its surface contacting the glass it is possible to measure the temperature distribution of the heating wall. Flow structure observation is carried out simultaneously at the opposite side of the minichannel.

Selected colour images of the heating foil in the measurement batch are presented in Fig. 3, the full batch was shown in [5]. When the current supplied to the 
heating foil increases gradually (images from \#1 up to \#4), it causes the occurrence of boiling incipience. The "boiling front" is recognizable as the hue sequence pattern, which indicates gradual hue changes to the liquid crystals and then sharp hue changes in the liquid crystal layer. Out-of-sensitivity-range temperatures are shown in black. This phenomenon of the occurrence of nucleation hysteresis was discussed in [1-6]. When the heat flux continues to increase, a new hue sequence in the upper part of images appears. This occurs when developed nucleate boiling is in progress in the minichannel. Then the current supplied to the foil is gradually reduced (images \#5 and \#6). Under such conditions, mild hue changes in the direction opposite to the spectrum sequence are observed. As a result, heat transfer returns to forced single phase convection. Images taken when the heat flux had been supplied to the foil were used for further analysis.

\subsection{Evaluation of the accuracy of heating foil} temperature measurements with liquid crystal thermography

Error estimation is conducted in accordance with the principles of measurement accuracy analysis [7]. Rootmean-square error is adopted as a measure of error quantity. Root-mean-square errors are computed as the roots of the sum of the second powers of the products of function partial derivatives, with respect to an external parameter occurring in a direct measurement, by the mean error of a given parameter measurement.

The course of calculations used for the evaluation of the accuracy of heating foil temperature measurements with liquid crystals thermography is presented in [1]. Similarly to $[1,8]$, it is assumed that the mean temperature error for the single point, determined on the basis of hue in calibration experiment, amounts to:

$$
\begin{aligned}
\sigma & =\frac{1}{P} \sum_{i=1}^{P} \sigma_{i} \\
& =\frac{1}{P} \sum_{i=1}^{P} \sqrt{\left(\frac{\partial T\left(\text { hue }_{i}\right)}{\partial h u e_{i}} \Delta h u e\right)^{2}+(2 S E E)^{2}+\left(\Delta T_{L}\right)^{2}}
\end{aligned}
$$

where: $i-$ measurement point; $P$-number of measurement points, $T$-temperature, $\Delta$ hue - error in hue determination for a recorded and processed image; it is assumed it equals double standard deviation, determined on the basis of the data for sample surface image in calibration experiment; $S E E$ - standard estimate of error in the fit of the calibration curve, determined with the use of the method of least squares, in accordance with formula:
$S E E=\sqrt{\frac{\frac{1}{P} \sum_{i=1}^{P}\left(T\left(\text { hue }_{i}\right)-T_{i}\right)^{2}}{P-m-n}}$

where: $m$ - the order of polynomial approximating a calibration curve, $m=16 ; n$-derivative order, $n=1 ; \Delta T_{L}$ absolute error in the measurement of liquid temperature during calibration.

Detailed calculations are presented in [9]. Absolute error in the measurement of liquid temperature at the minichannel inlet and outlet during calibration $\Delta T_{L}$ consists of two errors: the one resulting from signal processing by acquisition cards included in the measurement data acquisition station (incorporating the errors in the thermocouple card and in the station with 16-bit analogue-todigital converter) and the thermocouple sensor errors. The absolute thermocouple sensor errors result from the error in thermocouple calibration and the error in the measurement with a thermocouple. After calculations, the error resulting from signal processing by acquisition cards included in the measurement data acquisition station amounting to $69.974 \mu \mathrm{V}$ was obtained. In addition, the following results were calculated: the error in thermocouple calibration$6.582 \mu \mathrm{V}$, the error in the measurement using the thermocouple $-0.763 \mu \mathrm{V}$ and then thermocouple sensor errors-6.626 $\mu \mathrm{V}$. Subsequent calculations led to $\Delta T_{L}=70.287 \mu \mathrm{V}$. On the basis of thermocouple temperature measurement range (type $\mathrm{K}$ thermocouple, measurement range up to $1,100{ }^{\circ} \mathrm{C}$ ), and in the temperature range of the thermocouple card (from 0 to $100 \mathrm{mV}$ ) $1 \mathrm{~K}$ was obtained, corresponding to $90.91 \mu \mathrm{V}$. Therefore, $\Delta T_{L}$ corresponds to the error in temperature measurement $\Delta T_{L}=0.77 \mathrm{~K}$.

Sample results of calculations in the error analysis for the conducted experiment are as follows: $P=115$ calibration points: $\Delta h u e=1.45 ; \quad S E E=0.13 \mathrm{~K}$ and $\Delta T_{L}=0.77 \mathrm{~K}$ [1]. After calculations according to formula (1), $\Delta \sigma=0.86 \mathrm{~K}$.

\section{Void fraction determination}

\subsection{Visualisation of two phase flow structures}

The analyses of the flow structure were based on the monochrome images of flow structures with an SLR camera of heating foil, obtained on the side contacting fluid flowing in a minichannel. They were processed using Corel graphics software. After the photos had been binarized, the analysis of phase volumes was developed using special software-Techsystem Globe. The software allowed the determination of the areas of the two phases and/or 


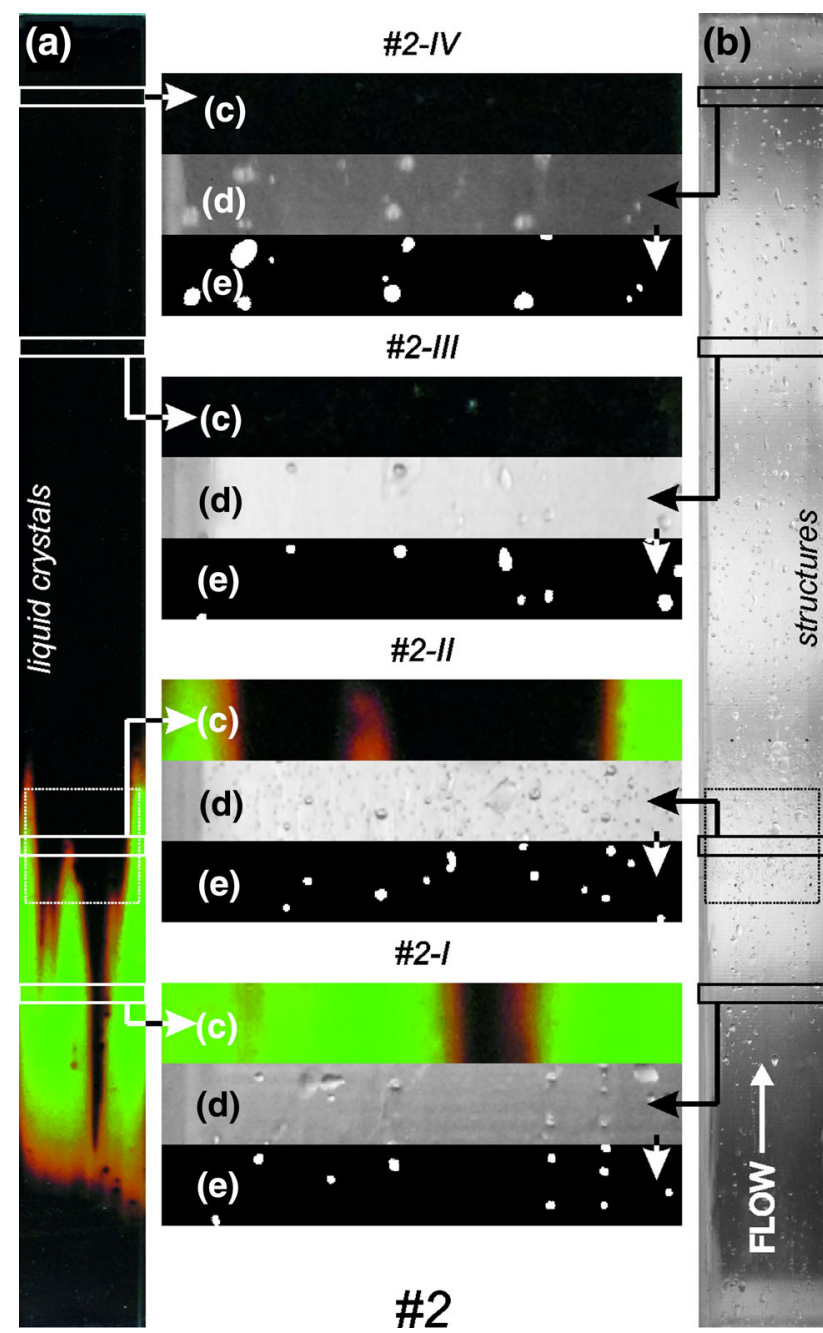

Fig. 4 Selected images for setting \#2: (a) colour image of the heating foil, $(b)$ greyscale image of flow structure image, $(c, d, e)$ images of cross sections: colour image of the heating foil $(c)$, two-phase flow structure images: real $(d)$ and binarized $(e)$; white colour refers to the vapour, and the black colour represents the liquid

percentage of the defined phase. Methodology of the conversion of greyscale image into monochrome image is presented in detail in [9]. In evaluation, the absolute error of the void fraction was assumed to be equal to the area (point) comprising $0.0064 \mathrm{~mm}^{2}$; it results from the resolution of the image taken by a digital camera $[4,9]$.

\subsection{Void fraction determination methodology}

Four initial settings (from \#1 up to \#4, Fig. 3) for increasing heat flux supplied to the heating surface of the foil were selected for analysis. Figure 4 shows a sample pictures for setting \#2 with marked cross-sections (Fig. 4a, b), colour image of cross sections (Fig. 4c), two-phase flow structure images: real (Fig. 4d) and binarized (Fig. 4e). Four cross-sections of the selected images (size $5 \mathrm{~mm} \times 40 \mathrm{~mm}$ ), were placed at the distance of $90 \mathrm{~mm}(\mathrm{I})$,

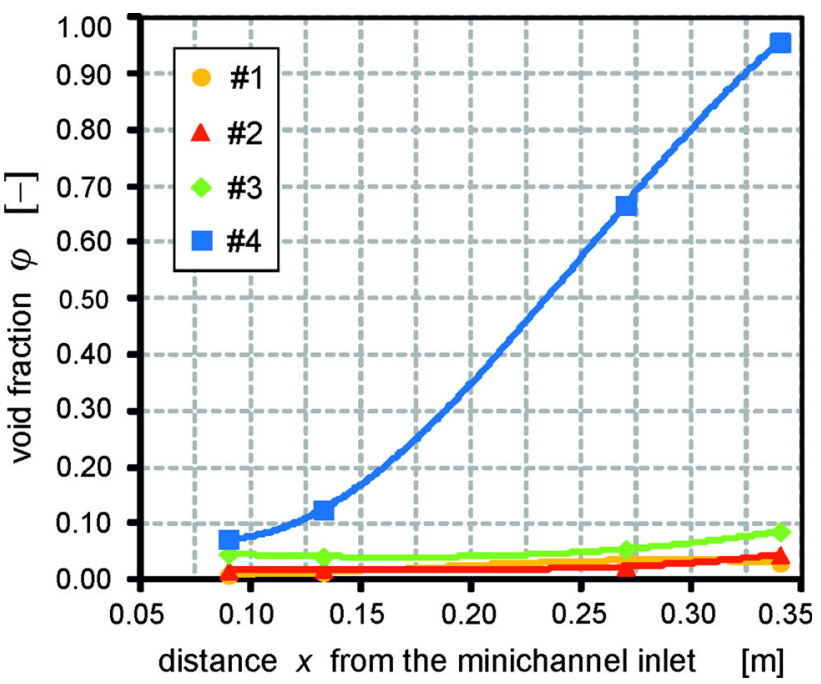

Fig. 5 Void fraction dependence along the minichannel length for selected cross sections for settings from \#1 up to \#4, parameters as for Fig. 3

$133 \mathrm{~mm}$ (II), $270 \mathrm{~mm}$ (III) and $336 \mathrm{~mm}$ (IV) from the inlet to the minichannel. The void fraction was determined according to the following formula [1-5]:

$\varphi=\frac{V_{v}}{V_{L}+V_{v}}=\frac{A_{v} \delta_{M}}{\left(A_{L}+A_{v}\right) \delta_{M}}=\frac{A_{v}}{A_{L}+A_{v}}$

where: $V$-volume, $A$-cross section area, $\delta$-depth.

In our notation, the subscripts referring to the glass pane, the heating foil, the liquid, the vapour and the minichannel are denoted with letters: $G, F, L, v$ and $M$, respectively.

Liquid and vapour cross-section areas were obtained from phase image analysis performed by means of Techsystem Globe software. The results are presented in the Fig. 5 as void fraction dependence along the minichannel length.

\section{Mathematical model}

\subsection{Problem formulation}

In the mathematical model the investigations took into account the dimension along the flow direction $(x)$, but the dimension perpendicular to it $(y)$ was related to the test section composed of the glass pane, heating foil and minichannel with cooling liquid. Further considerations are focused on the central part of the minichannel (along its length) so that the physical phenomena occurring on outer sides of the minichannel have no influence on thermodynamical parameters in the region studied.

Subcooling liquid at a temperature lower than the saturation temperature enters the minichannel and it is heated by the heating foil during its flow. We assume for both 
stationary and laminar flows $(R e=880)$ that velocity vector is parallel to the minichannel length so it has only one non-zero component $u=u(y)$. The component $u(y)$ is approximated by the following roof function:

$u(y)=\frac{u_{\max }}{\delta_{M}}\left(\delta_{M}-\left|2 y-\delta_{M}\right|\right)$

where $u_{\max }$ is equal to the doubled average velocity. The energy equation, exclusively for the liquid phase, has the following form:

$\nabla^{2} T_{L}=\frac{c_{L} \rho_{L}}{\lambda_{L}} u(y) \frac{\partial T_{L}}{\partial x}$

where: $\nabla^{2}=\frac{\partial^{2}}{\partial x^{2}}+\frac{\partial^{2}}{\partial y^{2}}, c$ - specific heat, $\rho$-density, $\lambda-$ thermal conductivity.

The following boundary conditions were specified for the Eq. (5):

temperature of the liquid in the inlet and outlet of the minichannel equals $T_{\text {in }}$ and $T_{\text {out }}$, respectively, i.e.

$T_{L}(0, y)=T_{\text {in }} \quad$ for $0 \leq y \leq \delta_{M}$

$T_{L}(L, y)=T_{\text {out }} \quad$ for $0 \leq y \leq \delta_{M}$

temperature of the liquid and the foil at the interface are equal:

$$
\begin{gathered}
T_{L}(x, 0)=\left\{\begin{array}{lll}
T_{F}(x, 0), & \text { if } & T_{F}(x, 0)<T_{\text {sat }}(x) \\
T_{\text {sat }}(x), & \text { if } & T_{F}(x, 0) \geq T_{\text {sat }}(x)
\end{array}\right. \\
\text { for } 0 \leq x \leq L
\end{gathered}
$$

where $T_{\text {sat }}$ is the saturation temperature dependent on the pressure, which changes linearly along the minichannel;

the two-phase mixture per unit volume in minichannel contains vapour phase and liquid phase in proportion $\varphi$ and $(1-\varphi)$, respectively. We assumed that the same proportion of vapour and liquid phases refers to any cross-sectional area of the minichannel and then to the heat exchange surface [10]. Hence, all heat generated by the heating foil is transferred to liquid phase proportionally to the void fraction

$\lambda_{L} \frac{\partial T_{L}}{\partial y}=\lambda_{F}(1-\varphi(x)) \frac{\partial T_{F}}{\partial y} \quad$ for $\quad y=0 \quad$ and $\quad 0 \leq x \leq L$

Stationary heat transfer in the glass pane and heating foil can be described by the following differential equations:

$$
\begin{array}{lll}
\text { (a) for glass } \nabla^{2} T_{G}=0 & \text { (b) for foil } \quad \nabla^{2} T_{F}=-\frac{q_{V}}{\lambda_{F}}
\end{array}
$$

where $q_{V}$ means volumetric heat flux. For these equations we assume following boundary conditions at the foil-glass interface:

$T_{F}\left(x_{i},-\delta_{F}\right)=T_{G}\left(x_{i},-\delta_{F}\right)=T_{i}$

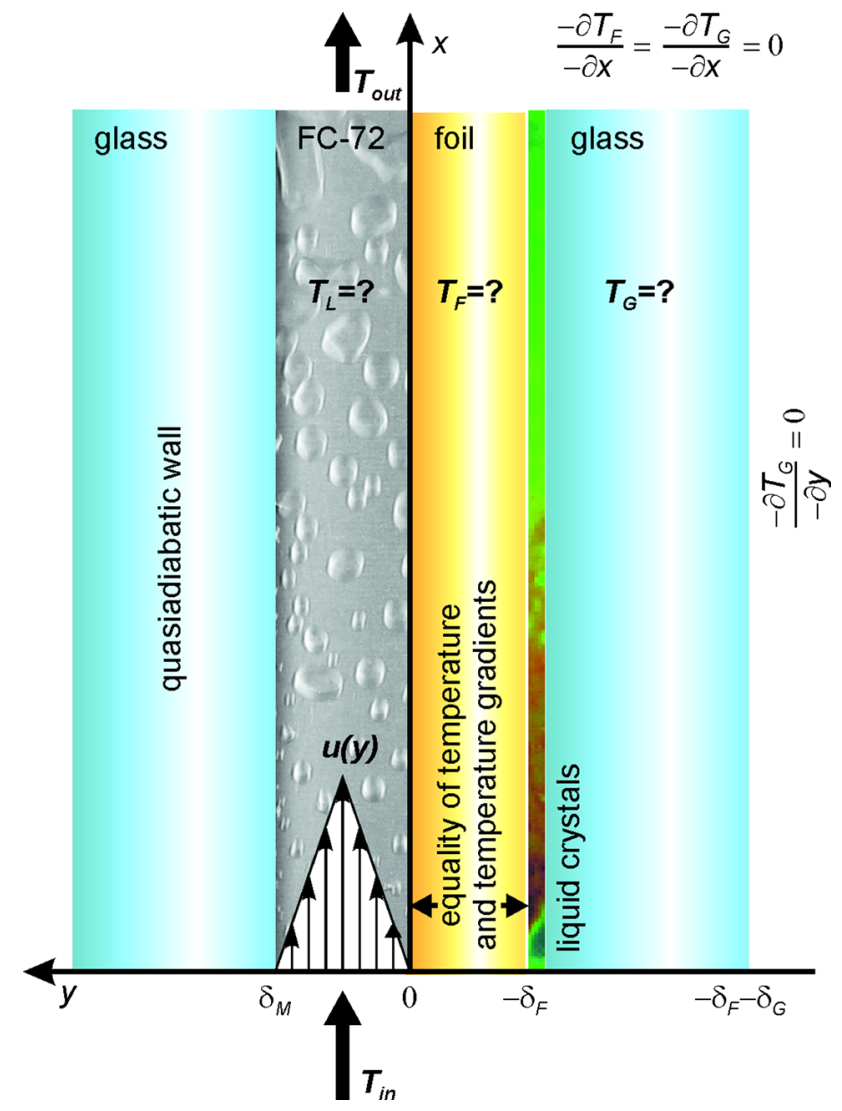

Fig. 6 Diagram of the boiling liquid flow in the minichannel, with the adapted boundary conditions (pictorial view, not to scale)

$\lambda_{F} \frac{\partial T_{F}}{\partial y}=\lambda_{G} \frac{\partial T_{G}}{\partial y} \quad$ for $y=-\delta_{F} \quad$ and $\quad 0 \leq x \leq L$

where $T_{i}$ means the measurements obtained with liquid crystal thermography at the foil-glass contact at discrete points $\left(x_{i}-\right.$ $\delta_{F}$ ). To complete specification of the functions $T_{F}$ and $T_{G}$ we assume that the outer surfaces of the minichannel are thermally isolated. Figure 6 contains the geometry and prescribed conditions of the considered problem.

\subsection{Trefftz method}

Equation (5) subject to prescribed boundary conditions (6)(9) will be solved by the Trefftz method $[6,10,11]$. In this method the unknown solution of a differential equation is approximated with a linear combination of the functions (so called Trefftz functions) which satisfy the governing equation. The unknown coefficients are determined by matching the boundary conditions. Before numerical calculations we have to generate Trefftz functions corresponding to Eq. (5) with roof-shaped velocity of the liquid, $u(y)$. The unknown solution to Eq. (5) is expanded in Taylor series, around the point (0.0): 


$$
\begin{aligned}
T_{L}(x, y)= & T_{L}(0,0)+\frac{\partial T_{L}}{\partial x} x+\frac{\partial T_{L}}{\partial y} y+\frac{1}{2 !} \frac{\partial^{2} T_{L}}{\partial x^{2}} x^{2} \\
& +\frac{2}{2 !} \frac{\partial^{2} T_{L}}{\partial x \partial y} x y+\frac{1}{2 !} \frac{\partial^{2} T_{L}}{\partial y^{2}} y^{2}+\frac{1}{3 !} \frac{\partial^{3} T_{L}}{\partial x^{3}} x^{3}+\ldots
\end{aligned}
$$

From Eq. (5) we get the dependence concerning the second partial derivative $\frac{\partial^{2} T_{L}}{\partial y^{2}}$

$\frac{\partial^{2} T_{L}}{\partial y^{2}}=\frac{c_{L} \rho_{L}}{\lambda_{L}} u(y) \frac{\partial T_{L}}{\partial x}-\frac{\partial^{2} T_{L}}{\partial x^{2}}$

which we substitute into Eq. (13) and after some algebraic manipulations the unknown solution $T_{L}$ has the following representation

$T_{L}(x, y)=\sum_{n=1}^{\infty} b_{n} v_{n}(x, y)$

where polynomials $v_{n}(x, y)$ are the desired Trefftz functions satisfying the energy Eq. (5) exactly.

Next, we approximate the solution $T_{L}$ with finitely many $v_{n}(x, y)$ 's which gives

$T_{L}(x, y) \approx \sum_{n=1}^{N} b_{n} v_{n}(x, y)$

The unknown coefficients of this expansion, $b_{n}$ will be selected to make the approximant $T_{L}$ best match (in variational sense) the boundary conditions (6)-(9). In terms of computations, by proper choice of $b_{n}$ 's one should minimize the functional $J$ that describes mean square error between function $T_{L}$ and prescribed boundary condition (6)-(9). The functional has the following form

$$
\begin{aligned}
J= & \int_{0}^{\delta_{M}}\left(\sum_{n=1}^{N} b_{n} v_{n}(0, y)-T_{\text {in }}\right)^{2} d y+\int_{0}^{\delta_{M}}\left(\sum_{n=1}^{N} b_{n} v_{n}(L, y)-T_{\text {out }}\right)^{2} d y \\
& +\int_{0}^{L}\left(\sum_{n=1}^{N} \lambda_{L} b_{n} \frac{\partial v_{n}(x, 0)}{\partial y}-\lambda_{F}(1-\varphi(x)) \frac{\partial T_{F}(x, 0)}{\partial y}\right)^{2} \\
& d x+\int_{0}^{L}\left(\sum_{n=1}^{N} b_{n} v_{n}(x, 0)-T_{L}(x, 0)\right)^{2} d x
\end{aligned}
$$

Finally we get the solution $T_{L}$ which satisfies the energy Eq. (5) exactly and the boundary conditions approximately. In the like manner we can obtain the solutions of Eqs. (10a and b) using Trefftz functions (harmonic polynomials) suitable for Laplace Eq. [11]. Trefftz method allows to determine two-dimensional distributions of temperature in the foil and in the glass as well as their gradients along the minichannel.

\section{Equalizing calculus}

Application of equalizing calculus means a choice of such corrections $\varepsilon_{i}$ for measurements $T_{i}$ that for the corrected measurements $T_{i}^{\text {corr }}$ the following condition is satisfied

$T_{i}^{\text {corr }}=T_{i}+\varepsilon_{i}$

Corrections $\varepsilon_{i}$ have normal distribution with the mean value equal to zero and finite variance equal to $\sigma_{i}^{2},[12,13]$, where errors $\sigma_{i}$ are defined by the Eq. (1). For $P$ of independent measurements the density function for corrected measurement results $T_{1}^{\text {corr }}, T_{2}^{\text {corr }}, \ldots, T_{P}^{\text {corr }}$ (and whereby corrections $\left.\varepsilon_{1}, \varepsilon_{2}, \ldots, \varepsilon_{P}\right)$ is delineated as the product of the density function for normal distribution i.e.

$\Phi\left(\varepsilon_{1}, \varepsilon_{2}, \ldots, \varepsilon_{P}\right)=\frac{1}{(2 \pi)^{0.5 P}\left(\sigma_{1} \sigma_{2} \ldots \sigma_{P}\right)} \exp \left[-\frac{1}{2} \sum_{i=1}^{P}\left(\frac{\varepsilon_{i}}{\sigma_{i}}\right)^{2}\right]$

Function $\Phi$ is called the likelihood function. Determination of corrections $\varepsilon_{i}$ leads to the determination of the maximum for likelihood function $\Phi$. Corrections determined by this method are always compliant and asymptotically efficient, i.e. when $P$ increases, the estimation result approaches the real value, whilst estimation variance will be as low as possible.

Function $\Phi$ is the highest when $\sum_{i=1}^{P}\left(\frac{\varepsilon_{i}}{\sigma_{i}}\right)^{2} \rightarrow$ min. If this is the case (when $\varepsilon_{i}$ have assumed normal distribution) the maximum likehood function method leads to the same results as the method of least squares. When applying the eqaulizing calculus in Trefftz method, the corrected value of foil temperature $T_{F}^{\text {corr }}$ should be determined to meet the following condition:

$T_{F}^{c o r r}\left(x_{i}, \delta_{G}\right)-T_{i}^{c o r r}=0$

The determination of the maximum of the likehood function $\Phi$, Eq. (19), assuming condition (20), leads to the minimalization of the Lagrange function:

$\Omega=\sum_{i=1}^{P}\left(\frac{\varepsilon_{i}}{\sigma_{i}}\right)^{2}+2 \sum_{i=1}^{P} \omega_{i}\left(T_{F}^{c o r r}\left(x_{i}, \delta_{G}\right)-T_{i}^{\text {corr }}\right) \rightarrow \min$

where $\omega_{i}$ were Lagrange multipliers.

Having corrected temperature measurements, we recalculated measurement errors $\sigma_{i}^{\text {corr }}$ according to the error propagation law [12,13]. As expected, equalizing calculus smoothed the measurement data and reduced the errors, see Fig. 8. Owing to known values of $T_{i}^{\text {corr }}$, it was possible to recalculate approximated temperatures of glass, foil (as described in [6]) and liquid, denoted $T_{G}^{\text {corr }}, T_{F}^{\text {corr }}$ and $T_{L}^{\text {corr }}$, respectively. 


\section{Results}

Numerical calculations were performed for experimental data and they concerned forced flow of the cooling liquid FC-72 through asymmetrically heated vertical

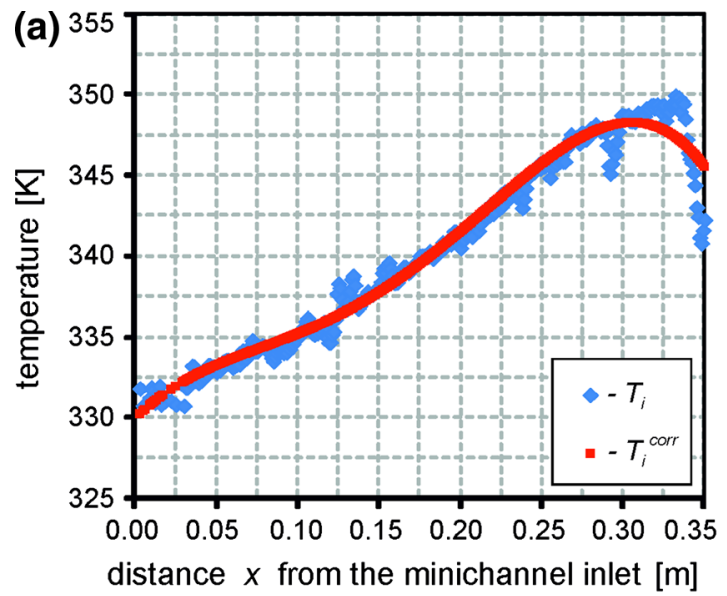

minichannel. Calculations were conducted for settings from \#1 up to \#4, with all parameters of the experiment given in Fig. 3. Settings \#1 and \#2 apply to boiling incipience, setting \#3 covers the interim status; setting \#4 applies to the developed boiling. The void fraction

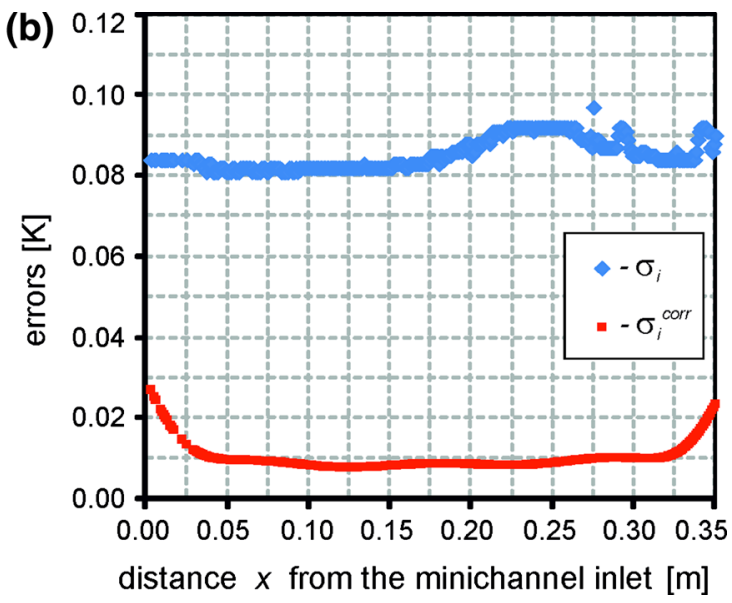

Fig. 7 a Temperature measurements $T_{i}$ and corrected measurements $T_{i}^{\text {corr }} ; \mathbf{b}$ measurement errors $\sigma_{i}$ and corrected measurement errors $\sigma_{i}^{\text {corr }}$, data for setting \#4

(a)

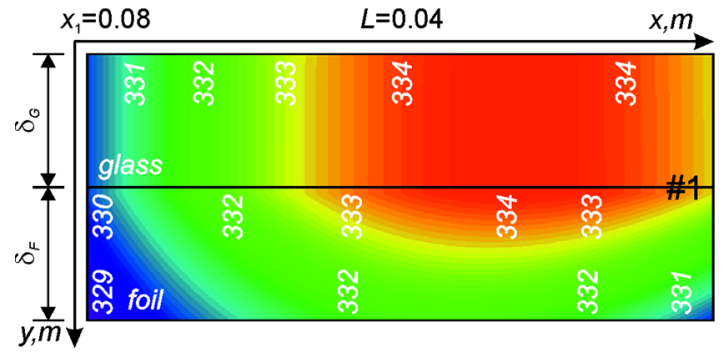

(c)

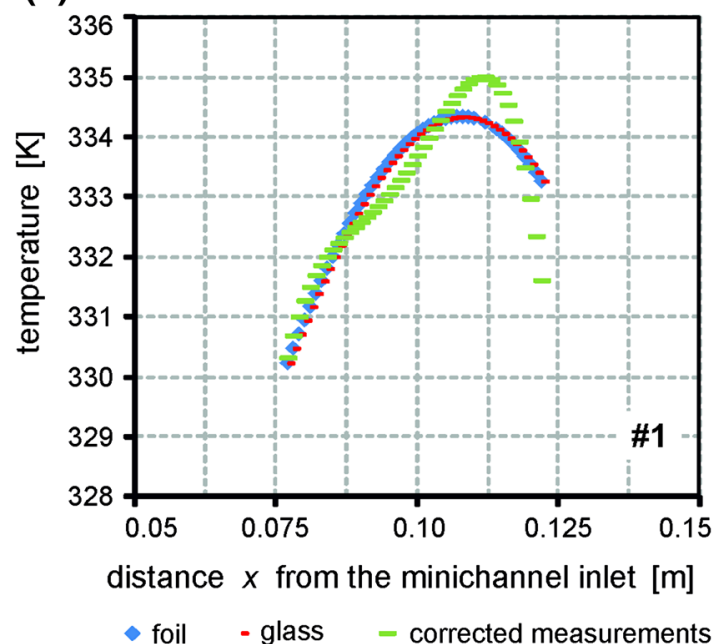

Fig. 8 a Two dimension temperature distributions of the glass pane and the heating foil, both obtained with Trefftz method for corrected measurements, b corrected temperatures of the corrected (b)

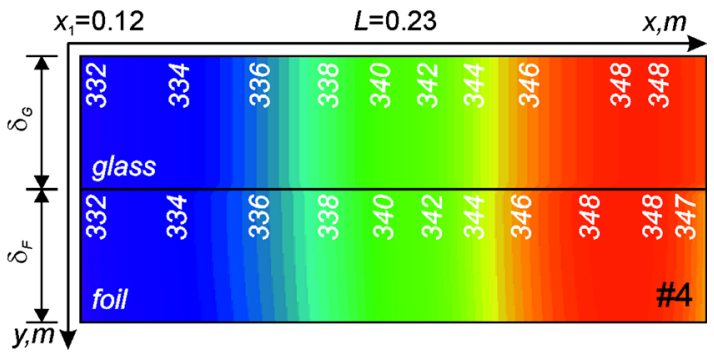

(d)

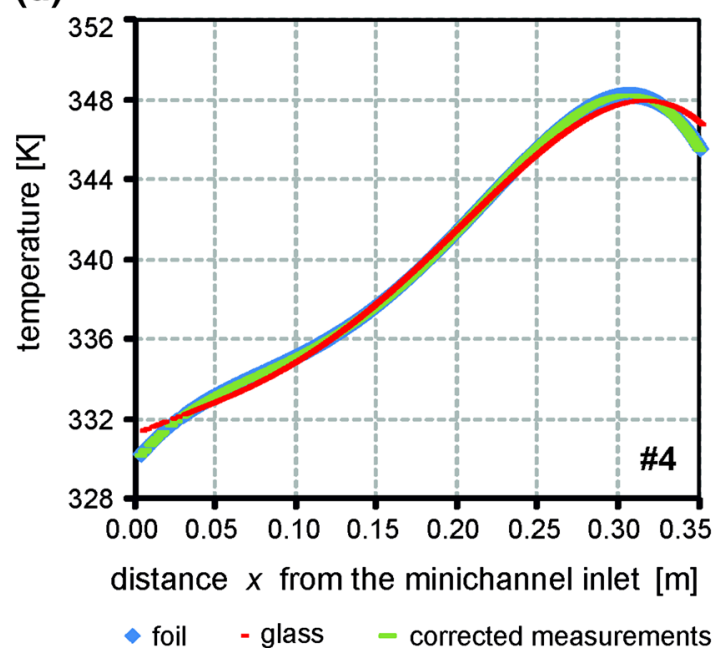

measurements (green), glass pane (red) and foil (blue) at the glassfoil contact, data for settings \#1 and \#4 (colour figure online) 
(a) $x_{1}=0.08$

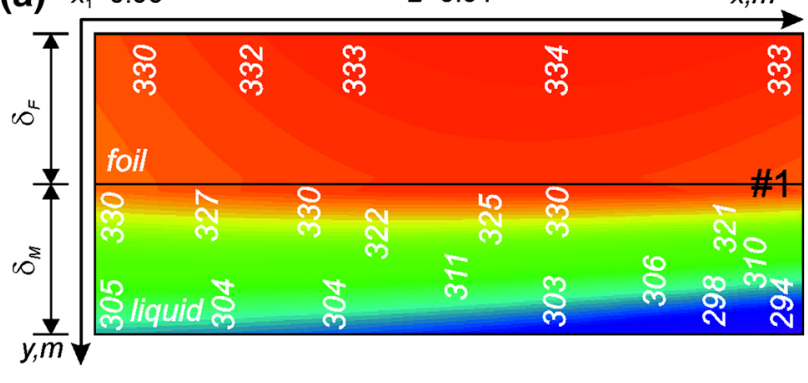

(b)

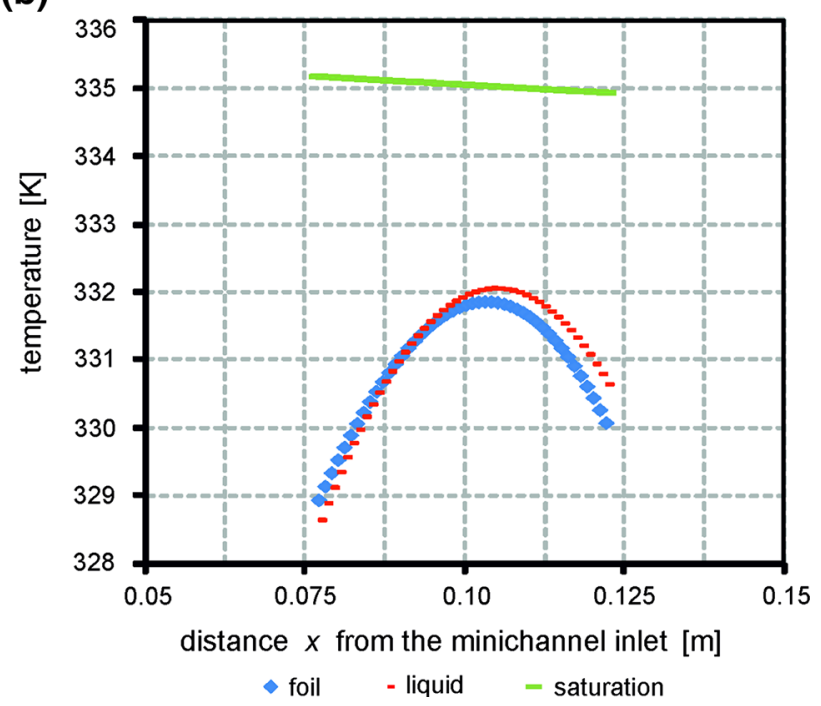

Fig. 9 a Two dimension temperature distributions of the heating foil and of the liquid, both obtained with Trefftz method, b corrected temperatures of the foil (blue), of the liquid (red) and saturation (green) at the foilliquid contact as a function of distance from the inlet to the channel. Setting \#1 (colour figure online)

described in detail in 2.2 and refereed to the settings under consideration, was approximated with a third degree polynomial as shown in Fig. 5.

At first, corrected measurements $T_{i}^{\text {corr }}$ were determined using equalizing calculus, Fig. 8b. Sample results obtained for setting \#4 are presented in Fig. 7a. Using the equalizing calculus, on the basis of the error propagation, law corrected errors $\sigma_{i}^{\text {corr }}$ were computed; their values are considerably lower than errors $\sigma_{i}$ calculated from (1), Fig. $7 \mathrm{~b}$.

Then computations involved the use of Trefftz method for finding two-dimensional temperature distribution in the glass pane and the heating foil. In the next step temperatures were calculated in the liquid phase. We designed approximate temperature distributions $T_{G}^{c o r r}, T_{F}^{c o r r}$ and $T_{L}^{c o r r}$ to be polynomials of the same degree. Fig. 8a presents twodimensional temperature distribution in the heating foil and the glass pane, both obtained by Trefftz method for corrected measurements $T_{i}^{\text {corr }}$. Either of the approximants satisfies the assumed conditions (11) and (12), with a relatively high accuracy, Fig. 8b. Similarly, Fig. 9a and Fig 10a shows
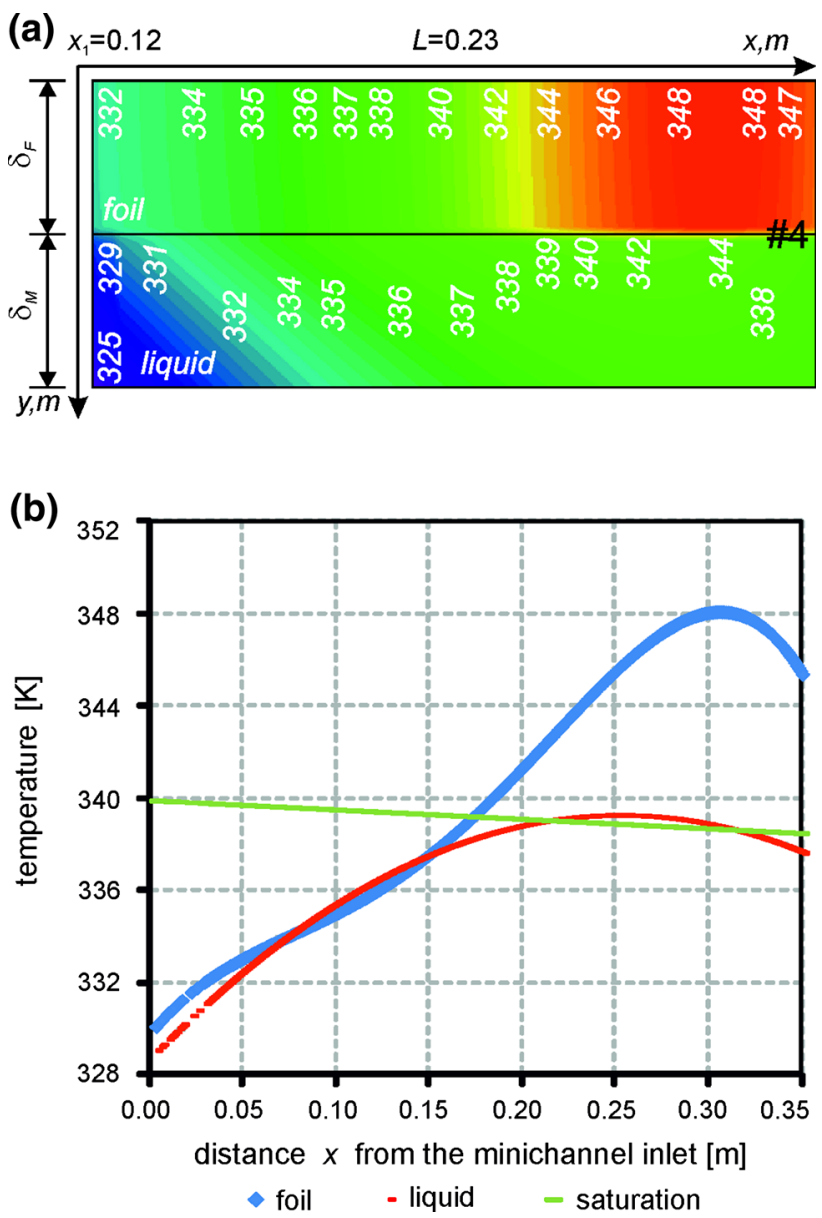

Fig. 10 a Two dimension temperature distributions of the heating foil and of the liquid, both obtained with Trefftz method, $\mathbf{b}$ corrected temperatures of the foil (blue), of the liquid (red) and saturation (green) at the foilliquid contact as a function of distance from the inlet to the channel. Setting \#4 (colour figure online)

temperature field in the heating foil and the flowing liquid. Figure 9 applies to the case when boiling incipience occurs (data for setting \#1), whilst Fig. 10 reflects the situation when developed boiling takes place (data for setting \#4). One can notice the characteristic shape of isotherms in the liquid (see Figs. 9a, 10a) which is due to modelling the velocity vector as a roof function. In Figs. $9 \mathrm{~b}$ and $10 \mathrm{~b}$ temperatures of the heating foil and the liquid at the foil-liquid interface are compared to saturation temperature. In [10] similar research, assuming a parabolic liquid velocity profile, was conducted for bubbly and bubbly-slug flow.

The known liquid and foil temperature distributions allowed calculating heat transfer coefficient $\alpha$ at the foilglass contact points from the Robin boundary condition [14].

$-\lambda_{F} \frac{\partial T_{F}^{c o r r}(x, 0)}{\partial y}=\alpha(x)\left(T_{F}^{c o r r}(x, 0)-T_{a v e}(x)\right)$.

Two options for computing liquid temperature were considered. In the first approach the averaged temperature 

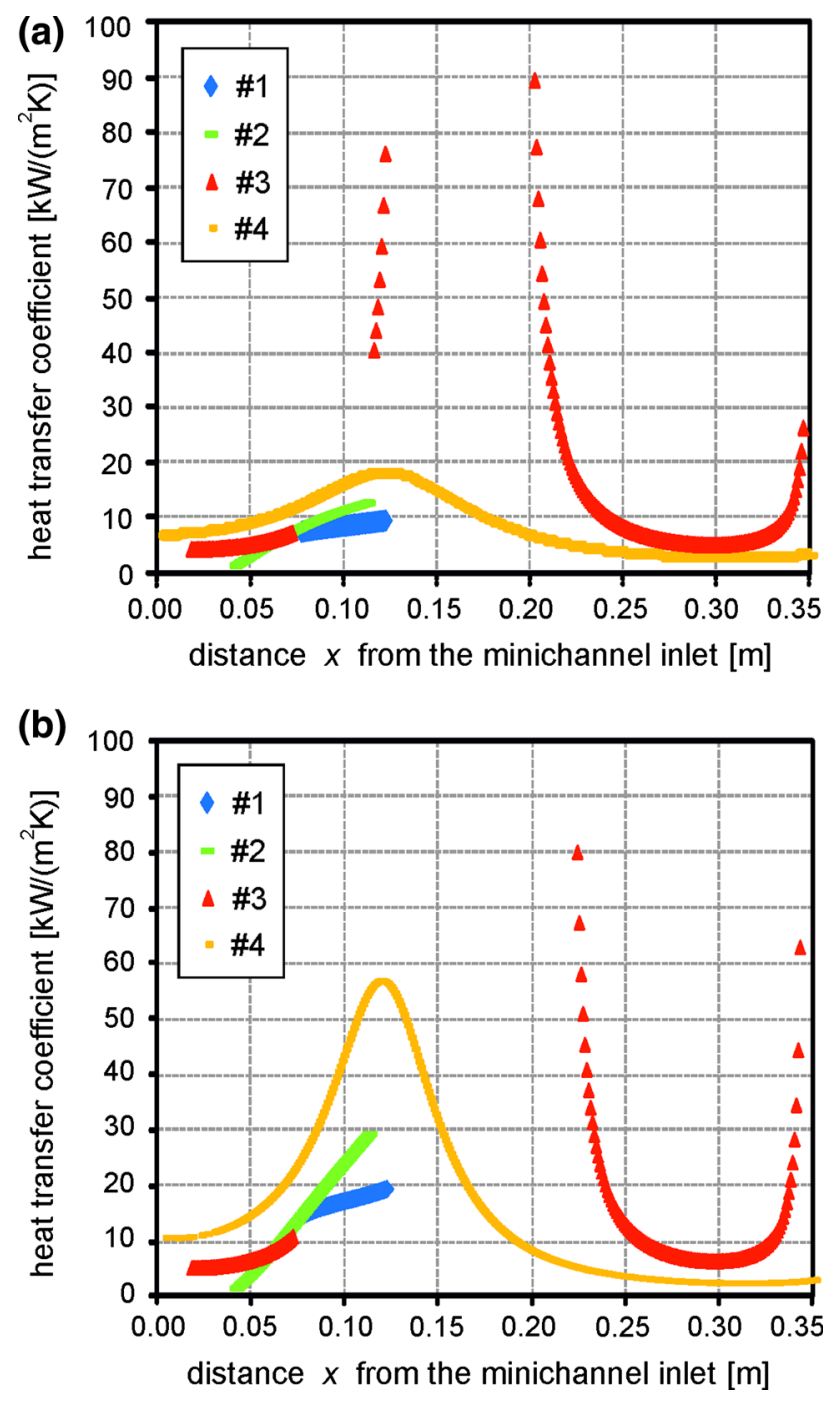

Fig. 11 Heat transfer coefficient as a function of distance from the inlet to the minichannel determined in the reference temperature: a from Eq. (23a), b from Eq. (23b)

across the whole width of the minichannel were assumed as reference temperature $T_{\text {ave }}$, Eq. (23a). Since the liquid reaches its maximal velocity along the axis of the minichannel, Eq. (2), in the second approach we took average temperature at points from $x=0$ to $x=0.5 \delta_{M}$ as reference temperature $T_{\text {ave }}$, Eq. (23b).

(a) $T 1_{\text {ave }}(x)=\frac{1}{\delta_{M}} \int_{0}^{\delta_{M}} T_{L}^{c o r r}(x, y) d y$

(b) $T 2_{\text {ave }}(x)=\frac{1}{0.5 \delta_{M}} \int_{0}^{0.5 \delta_{M}} T_{L}^{\text {corr }}(x, y) d y$

In the minichannel flow boiling, considerable heat transfer enhancement takes place at boiling incipience. It is observed as a sharp increase in the heat transfer coefficient at the foil-liquid contact, Fig. 11c. Similar results in flow boiling incipience were obtained in the study [4]. In the case of developed boiling, the heat transfer coefficient decreases as the void fraction increases. As far as the transition flow was concerned, the increase in the heat transfer coefficient was observed. For higher shares of the vapour phase in the two-phase mixture, heat transfer coefficient values will decrease.

\section{Conclusions}

The paper presents the approach to modelling laminar flow of the fluid during boiling incipience and developed boiling. When a mathematical model was formulated then the Trefftz method was used in order to solve it, however the validity of the solution is restricted to liquid phase only.

Special Trefftz functions had to be generated for the case of roof-shaped velocity of the liquid.

The proposed method based on Trefftz functions was successfully applied to the direct problem of finding temperature distribution in the glass pane. Moreover, this method turned to be suitable for the considered inverse problems: in the heating foil were we had a specified condition at one boundary and in the liquid where we assumed convective boundary condition in order to compute the heat transfer coefficient. The obtained numerical results show high compliance with physical assumptions of the model and have satisfactory accuracy.

Equilizing calculus applied to Trefftz method helped smooth the measurement data and reduce their errors.

In the minichannel flow boiling, considerable heat transfer enhancement takes place at boiling incipience. In the case of developed boiling, the heat transfer coefficient decreases as the void fraction increases. As far as the transition flow was concerned, the increase in the heat transfer coefficient was observed, but the data concerned a small range of the void fraction. For higher shares of the vapour phase in the two-phase mixture, heat transfer coefficient values will decrease. There are plans for indepth exploring transition and developed boiling area and future verification of the data.

Since the results based on Trefftz method turned to be promising, further research will be devoted to adapting the method to finding temperature distribution in the fluid which would include vapour phase as well as liquid phase.

Acknowledgments The research has been financially supported by the Polish Ministry of Science and Higher Education, Grant No. N N512 354037 for the years 2009-2014 and by the National Scientific Center granted on the basis of decision No. DEC-2013/09/B/ ST8/02825. 
Open Access This article is distributed under the terms of the Creative Commons Attribution License which permits any use, distribution, and reproduction in any medium, provided the original author(s) and the source are credited.

\section{References}

1. Piasecka M, Maciejewska B (2012) The study of boiling heat transfer in vertically and horizontally oriented rectangular minichannels and the solution to the inverse heat transfer problem with the use of the Beck method and Trefftz functions. Exp Thermal Fluid Sci 38:19-32

2. Piasecka M (2013) Heat transfer mechanism, pressure drop and flow patterns during FC-72 flow boiling in horizontal and vertical minichannels with enhanced walls. Int $\mathrm{J}$ Heat Mass Transf 66:472-488

3. Piasecka M (2014) The use of enhanced surface in flow boiling heat transfer in a rectangular minichannels. Exp Heat Transf 27:231-255

4. Piasecka M, Maciejewska B (2013) Enhanced heating surface application in a minichannel flow and use the FEM and Trefftz functions to the solution of inverse heat transfer problem. Exp Thermal Fluid Sci 44:23-33

5. Piasecka M (2013) An application of enhanced heating surface with mini-reentrant cavities for flow boiling research in minichannels. Heat Mass Transf 49:261-271
6. Hożejowska S, Piasecka M, Poniewski ME (2009) Boiling heat transfer in vertical minichannels. Liquid crystal experiments and numerical investigations. Int J Thermal Sci 48:1049-1059

7. Holman JP (1989) Experimental methods for engineers. McGraw-Hill, New York

8. Hay JL, Hollingsworth DK (1998) Calibration of micro-encapsulated liquid crystals using hue angle and a dimensionless temperature. Exp Thermal Fluid Sci 18:251-257

9. Piasecka M (2013) Determination of the temperature field using liquid crystal thermography and analysis of two-phase flow structures in research on boiling heat transfer in a minichannel. Metrol Meas Syst XX:205-216

10. Hożejowska S, Poniewski ME (2012) Application of the Trefftz method for determining the temperature field of the flowing boiling liquid in a minichannel. In: Proceedings of the 3th international conference on contemporary problems of thermal engineering, Gliwice, Poland (2012) Paper no 20

11. Grysa K, Ciałkowski MJ (2010) A sequential and global method of solving an inverse problem of heat conduction equation. J Theor Appl Mech 48:11-134

12. Brandt S (1999) Data analysis, statistical and computational methods for scientists and engineers. Springer, New York

13. Hożejowska S, Poniewski ME (2009) Various approaches to the calculation of minichannel flow boiling heat transfer. In: Proceedings of the 7th world conference on exp. Heat Transfer, Fluid Mechanics and Thermodynamics, Kraków, Poland, pp 1807-1814

14. Hożejowski L, Hożejowska S, Sokała M (2002) Evaluation of the Biot number with the use of heat functions. PAMM 1:349-350 